\title{
A STUDY OF RETINAL MORPHOLOGY AND FUNCTIONAL ASSESSMENT IN IDIOPATHIC CENTRAL SEROUS CHORIORETINOPATHY
}

\author{
Shubhra Das ${ }^{1}$ Dipak Bhuyan², Barun Garg ${ }^{3}$ \\ ${ }^{1}$ Associate Professor, Department of Ophthalmology, RIO, Guwahati Medical College and Hospital. \\ ${ }^{2}$ Associate Professor, Department of Ophthalmology, RIO, Guwahati Medical College and Hospital. \\ ${ }^{3}$ Postgraduate Trainee, Department of Ophthalmology, RIO, Guwahati Medical College and Hospital.
}

\section{ABSTRACT}

\section{BACKGROUND}

Idiopathic central serous chorioretinopathy is one of the common diseases of young adults characterised by an idiopathic, serous, neural, retinal detachment almost always in macular region. Diagnostic modalities such as FFA and OCT are used for confirmation of diagnosis of the disease.

The aim of this study is to evaluate characteristic retinal findings including functional parameters using various diagnostic modalities.

\section{MATERIALS AND METHODS}

This is a prospective study done at a Tertiary Care Centre. Patients presenting as ICSC on fundus examination irrespective of age and sex were subjected to FFA and OCT for confirmation of diagnosis. Further Multifocal Electroretinogram (mfERG) was done to evaluate the functional assessment.

\section{RESULTS}

OCT parameter such as central subretinal fluid thickness correlates with visual acuity, but for functional assessment mfERG reflects the level of visual acuity more significantly than structural evaluation by OCT.

\section{CONCLUSION}

OCT is a safe tool for diagnosis and followup of the patients with ICSC and complements findings with mfERG.

\section{KEYWORDS}

ICSC, OCT, MfERG.

HOW TO CITE THIS ARTICLE: Das S, Bhuyan D, Garg B. A study of retinal morphology and functional assessment in idiopathic central serous chorioretinopathy. J. Evolution Med. Dent. Sci. 2017;6(17):1323-1326, DOI: 10.14260/Jemds/2017/287

\section{BACKGROUND}

Idiopathic Central Serous Chorioretinopathy (ICSC) is a common retinal disorder affecting healthy individuals between 20 and 45 years.(1) It is characterised by an idiopathic, serous, neural retinal detachment almost always in macular region.(2)

It is usually a self-limiting disease, as the sensory detachment resolves spontaneously in most of the cases with fairly good recovery of vision, but in patients with recurrent and chronic episodes it can lead to RPE dysfunction and significant vision loss. ${ }^{(2,3)}$

The diagnosis of ICSC is generally established by slit lamp biomicroscopy and confirmed by Fluorescein Angiography (FFA) with OCT assisting as complimentary tool for presence of subretinal fluid, retinal thickening and pigment epithelial detachment. Newer diagnostic modalities especially mfERG (Multifocal Electroretinogram)(4) allows us do functional assessment at different retinal locations simultaneously.

Financial or Other, Competing Interest: None.

Submission 20-01-2017, Peer Review 14-02-2017,

Acceptance 19-02-2017, Published 27-02-2017.

Corresponding Author:

Dr. Shubhra Das,

House Number-15,

Lachit Nagar,

Guwahati-781007,

Assam.

E-mail: barun_garg@yahoo.com

DOI: $10.14260 /$ jemds $/ 2017 / 287$

(c) $(1) \ominus$
The majority of cases resolve simultaneously over time, therefore most often the initial treatment of choice is observation; also most of the patients acquire good visual acuity.(5) Though cases of chronic ICSC can undergo laser photocoagulation(2) or photodynamic therapy.(6)

\section{The Aim of the Study is to-}

1. Identify characteristical retinal findings of ICSC on FFA and optical coherence tomography.

2. Identify characteristic functional retinal findings of ICSC by appropriate electrophysiological test (MfERG).

The present study was a prospective study conducted in a Tertiary Care Centre. A total of 50 cases of ICSC were taken up for the study.

\section{MATERIALS AND METHODS}

All patients presenting with complaints of diminution or central haziness of vision were thoroughly screened and those with suggestive present and past history were subjected to further investigations for diagnosis and study of ICSC. Diagnosed cases were enrolled irrespective of age, sex and clinical variation. The period of the study was one year.

\section{Inclusion Criteria}

Patients with a clinical diagnosis of ICSC who had no contraindications to FFA were included. Only cases which resolved completely were further statistically analysed. 


\section{Exclusion Criteria}

1. Conditions that may masquerade ICSC like VogtKoyanagi-Harada syndrome, posterior scleritis.

2. Media opacity that may impair image quality of OCT.

After enrolment, the cases were clinically evaluated by examination of visual acuity, slit lamp examination, posterior segment evaluation was done using $+90 \mathrm{D}$ and $+20 \mathrm{D}$ lens, and the patients were subjected to special investigations like FFA and OCT for confirmation of diagnosis.

Then the functional tests like mfERG were performed on Metrovision Monpack-2 electrophysiology machine manufactured by Metrovision, France using 61 scaled hexagons. Considering recent developments in technology and practice, revised guidelines for clinical multifocal electroretinography (2007 edition) proposed by the International Society for Clinical Electrophysiology of Vision (ISCEV) were followed in the undertaken study.

In addition to above-mentioned test, the following laboratory investigations were done to exclude any other underlying pathology and to clear the patient for FFA

1. Routine blood examination.

2. Random blood sugar and serum creatinine.

3. Routine examination of urine and stool.

4. Chest x-ray.

5. Mantoux test.

In this study no medical or surgical treatment was given, except for managing any coexisting disease. Here only adequate counselling and expectant observation for spontaneous resolution was advised.

Patients were followed up at 1, 3, 6 and 9 months. In each visit, they were examined under the same parameters except mfERG which was repeated at 9 months and only in those cases which resolved completely and were further statistically analysed.

\section{RESULTS AND OBSERVATIONS}

A total of 50 eyes from 50 patients attending the OPD were evaluated. The period of study was 1 year. Different results observed in the study are documented as follows -

The mean patient age in this study was 36.88 years (SD 7.577; range 22 - 55, median - 35). Fifty percent of the patients were in the age group of 30 - 39 years. Out of fifty newly diagnosed cases, $76 \%$ of them were male and $24 \%$ females with ratio of 3.17:1 in favour of males.

The mean duration of symptoms was 15 days with a minimum presentation of 1 day and maximum of 90 days. All patients had unilateral active disease of which right eye was affected in $46 \%$ and left eye in $54 \%$ cases. Only 2 cases (4\%) had a record of previous attacks.

The patients presented with diminution of vision (100\%), dark shadow in front of eye (50\%), distortion of objects (36\%), altered colour perception (8\%) and headache (12\%). Two female patients had ICSC at third trimester of pregnancy, but it completely resolved after delivery of baby.

\begin{tabular}{|c|c|c|c|}
\hline $\begin{array}{c}\text { Visual } \\
\text { Acuity } \\
\text { (VA) }\end{array}$ & $\begin{array}{c}\text { VA in } \\
\text { Decimal } \\
\text { System }\end{array}$ & $\begin{array}{c}\text { Number of } \\
\text { Eyes }\end{array}$ & $\begin{array}{c}\text { Percentage } \\
\text { (\%) }\end{array}$ \\
\hline $6 / 9$ & 0.67 & 12 & 24 \\
\hline $6 / 12$ & 0.50 & 5 & 10 \\
\hline $6 / 18$ & 0.33 & 19 & 38 \\
\hline $6 / 24$ & 0.25 & 7 & 14 \\
\hline $6 / 36$ & 0.17 & 4 & 8 \\
\hline $6 / 60$ & 0.10 & 3 & 6 \\
\hline \multicolumn{4}{|c|}{ Table 1. Visual Acuity of the Patients } \\
\hline
\end{tabular}

Amsler grid test elucidated metamorphopsia in $70 \%$ of patients, which was more pronounced in just outside nonseeing area, positive scotoma in $78 \%$ and micropsia in $4 \%$ of patients. Photostress recovery test was found to be prolonged in almost all cases for white light between 25 to 110 seconds. It was observed that more the defect in visual acuity more was the recovery time.

On fundus examination, a well-defined round to oval area of shallow elevation of retina in the macular area was noted with dull foveal reflex.

\begin{tabular}{|c|c|c|}
\hline $\begin{array}{c}\text { Fundus } \\
\text { Findings }\end{array}$ & $\begin{array}{c}\text { Number } \\
\text { of Eyes }\end{array}$ & $\begin{array}{c}\text { Percentage } \\
\text { (\%) }\end{array}$ \\
\hline $\begin{array}{c}\text { Sensory retinal } \\
\text { detachment }\end{array}$ & 48 & 96 \\
\hline Subretinal precipitates & 18 & 36 \\
\hline Drusen-like deposits & 6 & 12 \\
\hline PED & 7 & 14 \\
\hline Depigmented patches & 7 & 14 \\
\hline
\end{tabular}

Table 2. Table Showing Different Fundus Findings in ICSC Patients in Macular Area

Fundus Fluorescein Angiography (FFA) - all patients underwent FFA, out of which $86 \%$ had single leak, $8 \%$ had double leak and $6 \%$ had no leak. The most common pattern of leakage was ink-blot (80\%) followed by smoke stack (8\%), diffuse pattern $(8 \%)$ and minimal leak in $4 \%$ of the cases. Leaks were most commonly located in superior nasal quadrant (54\%) followed by inferior nasal (24\%).

OCT - it clearly demonstrated configuration of detached neurosensory retina and retinal pigment epithelium. All patients had serous retinal detachment with empty vaulted area of variable height (range: 58 - 930 um, mean - 316.2 um).

\begin{tabular}{|c|c|c|}
\hline & \multicolumn{2}{|c|}{ BCVA } \\
\hline OCT Parameter & Spearman Coefficient & P value \\
\hline Central SRF & -0.3275 & 0.0202 \\
\hline Central Foveal Thickness & -0.2116 & 0.1401 \\
\hline Table 3. Table showing Correlation of BCVA with OCT \\
Parameters \\
\hline
\end{tabular}

Non-parametric Spearman correlation was done between BCVA and OCT parameters, out of which central sub-retinal fluid and BCVA showed P value of 0.0202 indicating a strong negative correlation, which indicated that more the fluid accumulation worse was the vision.

The OCT was used to follow up cases, $20 \%$ of total cases resolved within one month, another $20 \%$ within 3 months, next $30 \%$ within 6 months and all cases resolved by 9 months. 
MfERG- in the current study Monpack-2 Electrophysiological unit was used for mfERG. The responses were recorded and three types of plots were made. Two and three dimensional charts were made on the basis of trace chart. The first negative trough (N1) and first positive peak (P1) were identified on the concentric rings. As ICSC mainly affects macular area, N1 and P1 wave amplitude and implicit time of first two rings that is $<2$ degrees and $2-5$ degrees were evaluated and correlated with BCVA and OCT parameters.

\begin{tabular}{|c|c|c|}
\hline & Affected Eye & Unaffected Eye \\
\hline P1 amplitude & & $1262.58 \pm 389.23$ \\
\hline$<2$ degrees & $663.66 \pm 264.34$ & $802.82 \pm 163.96$ \\
\hline $2-5$ degrees & $453.6 \pm 150.83$ & $43.18 \pm 4.38$ \\
\hline P1 implicit time & $54.95 \pm 8.02$ & $41.36 \pm 2.81$ \\
\hline$<2$ degrees & $46.79 \pm 5.24$ & $-916.68 \pm 174.17$ \\
\hline $2-5$ degrees & & $-718.62 \pm 138.19$ \\
\hline N1 amplitude & $-530.12 \pm 185.54$ \\
\hline$<2$ degrees & $-384.26 \pm 86.10$ & $20.65 \pm 1.62$ \\
\hline $2-5$ degrees & & $20.43 \pm 1.5$ \\
\hline N1 implicit time & $23.28 \pm 1.88$ & \\
\hline$<2$ degrees & $22.69 \pm 1.48$ & \\
\hline $2-5$ degrees &
\end{tabular}

There was significant difference between affected and normal uninvolved eye in both amplitude as well as implicit time. P1 and N1 amplitude were found to be much lower in macular area in affected eye as compared to unaffected eye, whereas P1 and N1 time was much higher in affected eye as compared to unaffected eye.

\begin{tabular}{|c|c|c|c|c|c|c|}
\hline & \multicolumn{2}{|c|}{ Central SRF Thickness } & \multicolumn{2}{|c|}{ Total Central Foveal Thickness } & \multicolumn{2}{|c|}{ BCVA } \\
\hline & $\begin{array}{c}\text { Spear- } \\
\text { man } \\
\text { Sigma }\end{array}$ & $\begin{array}{c}\mathbf{P} \\
\text { value }\end{array}$ & $\begin{array}{l}\text { Spear- } \\
\text { man } \\
\text { Sigma }\end{array}$ & $\begin{array}{c}P \\
\text { value }\end{array}$ & $\begin{array}{c}\text { Spear-man } \\
\text { Sigma }\end{array}$ & $\begin{array}{c}P \\
\text { value }\end{array}$ \\
\hline \multicolumn{7}{|l|}{ P1 amplitude } \\
\hline$<2$ degrees & -0.2906 & 0.0407 & -0.2458 & 0.0853 & 0.4866 & 0.0003 \\
\hline 2 - 5 degrees & -0.3936 & 0.0047 & -0.2830 & 0.0465 & 0.3975 & 0.0043 \\
\hline \multicolumn{7}{|l|}{ P1 implicit time } \\
\hline$<2$ degrees & 0.3220 & 0.8243 & 0.0983 & 0.4970 & -0.3332 & 0.0180 \\
\hline 2 - 5 degrees & 0.1082 & 0.4545 & 0.05259 & 0.7163 & -0.0241 & 0.8681 \\
\hline \multicolumn{7}{|l|}{ N1 amplitude } \\
\hline$<2$ degrees & -0.3591 & 0.0104 & -0.02895 & 0.0414 & 0.6631 & 0.001 \\
\hline 2 - 5 degrees & -0.1598 & 0.2677 & -0.1799 & 0.2113 & 0.2603 & 0.0679 \\
\hline \multicolumn{7}{|l|}{ N1 implicit time } \\
\hline$<2$ degrees & 0.1278 & 0.3766 & 0.06588 & 0.6494 & -0.3170 & 0.0249 \\
\hline 2 - 5 degrees & 0.03744 & 0.7963 & 0.02284 & 0.8749 & -0.4719 & 0.0005 \\
\hline \multicolumn{7}{|c|}{ Table 5. Table showing Correlation of mfERF Parameters with various OCT Parameters and BCVA } \\
\hline
\end{tabular}

\section{DISCUSSION}

In this study, all patients having ICSC underwent investigations and were confirmed by fundus fluorescein angiography and optical coherence tomography.

FFA used as the diagnostic test to confirm ICSC in 50 patients showed that $88 \%$ patients had unilateral leak, $2 \%$ had bilateral leak and $6 \%$ had no leak. It also demonstrated that $86 \%$ of the eyes had single leak and $4 \%$ had double leak. Most common pattern of leakage was found to be ink blot (80\%) followed by smoke stack. Literature shows that Spitznas and Huke,(7) and Gass( ${ }^{(8)}$ also made similar observations. Besides leaking points, $20 \%$ of cases had Pigment Epithelial Detachment (PED) in active eye and 8\% cases in the fellow eye. FFA helps in detecting PED underlying serous detachment, which is quite often difficult to appreciate clinically.
OCT offers an objective test for the quantitative evaluation of patients with ICSC, as it is able to track resolution of the sensory retinal detachment with more sensitivity than slit lamp examination with +90D lens. In the acute phase, the detached retina is thicker than adjacent retina at the border of the serous retinal detachment though the layered structure of retina was very well preserved. Similar observations were reported by Hee et al(9) and Lida et al.(10)

Non-parametric Spearman correlation analysis between Snellen's BCVA expressed as decimal system and OCT findings showed negative correlation between BCVA and central subretinal fluid thickness, but no significant correlation was found with central foveal thickness; therefore, it indicates central subretinal fluid thickness to be a more reliable indicator.

The OCT machine has $100 \%$ sensitivity for diagnosis and followup for patients. It also demonstrated that higher the 
central SRF thickness, more time was needed for resolution of ICSC. Yip et al showed similar results.(11)

MfERG provided objective topographic evaluation of macular function during active and resolved stages. Impairment in the conduction of electrical responses from photoreceptors and inner retinal layers as reflected by $\mathrm{N} 1$ and P1 wave respectively were in proportion to the amount of SRF in ICSC. Significant difference between the affected eye during acute stage and normal uninvolved eye in both amplitude as well as implicit time when $<2$ and 2 - 5 degrees area was considered for both P1 and N1 wave ( $<<0.0001$, unpaired ' $\mathrm{t}$ ' test with Welch correction). Studies by Chappelow and Marmor,(12) Aoyagi et al(13) also demonstrated similar results.

Significant positive correlation was found between BCVA and P1 amplitude $(<2$ and $2-5$ degrees area) and N1 amplitude $(<2$ degrees), but significant negative correlation was seen between BCVA when compared to P1 ( $<2$ degrees) and N1 implicit time ( $<2$ and 2 - 5 degrees area).

There was a significant negative correlation between central SRF thickness and P1 and N1 implicit time $(<2$ and 2 5 degrees area). Similar results were obtained by Yip et al(11) but they did not take into consideration the foveal threshold. So impairment in conduction of electrical responses from photoreceptors and inner retinal layers as reflected by $\mathrm{N} 1$ and P1 wave respectively in proportion to the amount of SRF in ICSC.

Therefore, it is observed that though MfERG and OCT can complement each other in the functional and anatomical assessments of patients with ICSC, but functional assessment using MfERG reflects the level of visual acuity more significantly than anatomical evaluation by OCT.

In the resolved stage MfERG parameters did not recover to normal level, even if SRF has resolved and visual acuity is restored to near normal level indicating some functional impairment at macula. The prognosis for resolution and visual recovery for patients with ICSC is excellent.

\section{CONCLUSION}

1. OCT is a safe tool for diagnosis and followup of the patients with ICSC and complements findings with mfERG.

2. Though mfERG and OCT can complement each other in the functional and anatomical assessments of patients with ICSC, but functional assessment using mfERG reflects the level of visual acuity more significantly than anatomical evaluation by OCT.

3. OCT parameter such as central subretinal fluid thickness to be a more reliable indicator than central foveal thickness in estimating the visual acuity.

\section{REFERENCES}

[1] Kitzmann AS, Pulido JS, Diehl NN, et al. The incidence of central serous chorioretinopathy in olmsted county, minnesota, 1980-2002. Ophthalmology 2008;115(1):169-73.

[2] Donald J, Gass M. Pathogenesis of disciform detachment of the neuroepithelium. American journal of ophthalmology 1967;63(3):587-615.

[3] Spitznas M. Pathogenesis of central serous retinopathy: a new working hypothesis. Graefes Archive for Clinical and Experimental Ophthalmology 1986;224(4):321-4.

[4] Dale EA, Hood DC, Greenstein VC, et al. A comparison of multifocal ERG and frequency domain OCT changes in patients with abnormalities of the retina. Documenta ophthalmologica 2010;120(2):175-86.

[5] Klein ML, Buskirk VEM, Friedman E, et al. Experience with nontreatment of central serous choroidopathy. Archives of Ophthalmology 1974;91(4):247-50.

[6] Piccolino FC, Eandi CM, Ventre L, et al. Photodynamic therapy for chronic central serous chorioretinopathy. Retina 2003;23(6):752-63.

[7] Spitznas M, Huke J. Number, shape, and topography of leakage points in acute type I central serous retinopathy. Graefe's archive for clinical and experimental ophthalmology 1987;225(6):437-40.

[8] Gass JD. Stereoscopic atlas of macular diseases: diagnosis and treatment. $4^{\text {th }}$ edn. St Louis, Mosby Inc 1997:52-70.

[9] Puliafito CA, Hee MR, Lin CP, et al. Imaging of macular diseases with optical coherence tomography. Ophthalmology 1995;102(2):217-29.

[10] Iida T, Yannuzzi LA, Spaide RF, et al. Cystoid macular degeneration in chronic central serous chorioretinopathy. Retina 2003;23(1):1-7.

[11] Yip YW, Fok AC, Ngai JW, et al. Correlation between functional and anatomical assessments by multifocal electroretinography and optical coherence tomography in central serous chorioretinopathy. Doc Ophthalmol 2010;120(2):193-200.

[12] Chappelow AV, Marmor MF. Multifocal electroretinogram abnormalities persist following resolution of central serous chorioretinopathy. Archives of ophthalmology 2000;118(9):1211-5.

[13] Aoyagi K, Kimura Y, Isono $H$, et al. Multifocal electroretinogram in central serous chorioretinopathy. Invest Ophthalmol Vis Sci 1998;39:S185. 